\title{
Hearing loss and strial microvascular pathology-towards unravelling the functional contribution of the blood- labyrinth barrier
}

\author{
Carola Y Förster ${ }^{1 *}$, Verena Scheper ${ }^{2,3}$ and Thomas Lenarz ${ }^{2,3}$ \\ ${ }^{1}$ Department of Anaesthesia and Critical Care, University Hospital Wuerzburg, Wuerzburg, Germany \\ ${ }^{2}$ Department of Otolaryngology, Hannover Medical School, Hannover, Germany \\ ${ }^{3}$ Cluster of Excellence 'Hearing4all', German Research Foundation, Germany
}

Disabling and permanent hearing loss affects more than $5 \%$ of the world's population as reviewed by a 2015 report by the World Health Organization (https://www.who.int/news-room/fact-sheets/ detail/deafnessand-hearing-loss). Hearing loss, mostly involving irreversible loss of sensory cells in the cochlea or loss of auditory neurons, is classified as sensorineural in more than $90 \%$ of cases. Hereby, the main external events leading to acquired hearing loss are noise trauma (noise-induced hearing loss NIHL) and ototoxic treatments as radiation therapy, aminoglycoside antibiotic treatment or platinum-based chemotherapy. Moreover, about $50 \%$ of cases have been identified to be caused by congenital deafness through more than 500 genetic mutations [1-3].

No FDA-approved drug therapies are available to protect or restore hearing. The main FDA-approved treatment for debilitating hearing loss is the cochlear implant, which, for an insertion of the electrode array into the cochlea, requires invasive surgery [4].

Ion homeostasis, transport of nutrients and systemic hormones to the inner ear is maintained by the blood-labyrinth barrier (BLB) in the cochlear lateral wall [1]. While these blood vessels of the inner ear are critical for normal function, they are however in the same time highly vulnerable to pathologic events that result in hearing and vestibular dysfunction. Despite these numerous critical roles of inner ear vasculature to enable and facilitate hearing, little is known of its normal homeostatic functions and how these are altered in disease.

As demonstrated for the blood-brain barrier (BBB) [5-8], maintaining the homeostasis of the central nervous system, different disease states can alter BLB physiology and increase its permeability to specific compounds; for example, inflammation caused by either local (acoustic trauma) [9] or systemic immunogenic stimuli alters uptake of aminoglycoside antibiotics [10] and diuretics.

Inflammation has been extensively demonstrated to generally lead to vasodilation, facilitating paracellular flux into the interstitial extracellular space. In the BLB, cells being tightly coupled by tight junctions, vasodilation apparently occurs without increased paracellular flux but does result in increased cochlear uptake of ototoxic aminoglycosides into the stria vascularis or of fluorophores into the perilymph [11-13].

Acoustic trauma may correspondingly affect the permeability of the BLB and through this subsequent influence drug uptake indirectly by inducing inflammation. Loud sounds hereby affect virtually all cochlear cell types and induce cochlear inflammation and through this, in turn, drug-induced cochleotoxicity [14-16]. Intriguingly, the synergistic ototoxicity of loud sound exposure and aminoglycosides appears not to be limited to simultaneous exposure. Acoustic trauma, even experienced days or weeks before aminoglycoside treatment, can even potentiate drug-induced hearing loss $[10,14]$, suggesting that cochlear inflammation persists for days after induction. Furthermore, although limited data exist, hypoxia also appears to induce inflammatory signaling cascades as it has been described for the BBB [17].

Age-related hearing loss (ARHL), also referred to as presbycusis, is the most common sensory loss in the aging individuum. Bilateral hearing loss occurrence doubles every 10 years over the age of 50 [18]. The future management of ARHL is thus a major social and socioeconomic challenge through the increased longevity of the population, especially as ARHL has been described to be positively correlated with cognitive impairment and dementia [19].

ARHL is most probably highly affected by the aging-induced alterations in BLB-integrity. In the cochlear lateral wall of rodents for example, the most commonly described finding with increasing age of the animals is a decrease in the area or volume of the stria vascularis. The underlying molecular pathological processes remain however poorly described. A comprehensive characterization of aging-induced compromise of BLB function is outstanding, to enable preventive and curative care.

Given the important role of the BLB and its alterations in the etiology of the different pathological processes leading to sensorineural hearing loss, more data characterizing cell biology and function of the BLB are needed to fully elucidate the properties of the BLB, to help unlock the potential for prevention and cure of hearing loss.

Both, BBB and BLB serve to provide protection to the delicate structures of the organs brain and inner ear, respectively by forming a dynamic but tight biological barrier. Dysfunction of either barrier, $\mathrm{BBB}$ or $\mathrm{BLB}$, is part of a broader spectrum of common disease. A more

${ }^{\star}$ Correspondence to: Carola Y Förster, Department of Anaesthesia and Critical Care, University Hospital Wuerzburg, Wuerzburg, Germany, Tel: +49 931201 30065, Fax: (0931)20130019; E-mail: foerster_c@ukw.de

Received: November 04, 2019; Accepted: November 20, 2019; Published: November 25, 2019 
thorough understanding of the role of the BLB in physiology and pathology of hearing could thus form the basis to a paradigm change and significant new cut point for the future treatment of hearing loss by (i) promoting a better understanding of molecular-biological causes of hearing disorders among basic researchers, (ii) translating this knowledge to pave the way for new clinical intervention, including pharmacological therapies, in hearing disorders, (iii) making an important overall contribution to reduce the burden related to hearing loss in Europe, and worldwide.

\section{References}

1. Nyberg S, Abbott NJ, Shi X, Steyger PS, Dabdoub A (2019) Delivery of therapeutics to the inner ear: The challenge of the blood-labyrinth barrier. Sci Transl Med 11: eaao0935. [Crossref]

2. O'Connell BP, Hunter JB, Haynes DS (2016) Current concepts in the management of idiopathic sudden sensorineural hearing loss. Curr Opin Otolaryngol Head Neck Surg 24: 413-419. [Crossref]

3. Kremer H (2019) Hereditary hearing loss; about the known and the unknown. Hear Res 376: 58-68. [Crossref]

4. Bootz F, Lenarz T (1992) Reconstructions in the head and neck area using a free latissimus dorsi flap. Laryngorhinootologie 71: 31-34.

5. Sebastiani A, Greve F, Golz C, Forster CY, Koepsell H, et al. (2018) RS1 (Rsc1A1) deficiency limits cerebral SGLT1 expression and delays brain damage after experimental traumatic brain injury. J Neurochem 147: 190-203. [Crossref]

6. Blecharz KG, Haghikia A, Stasiolek M, Kruse N, Drenckhahn D, et al. (2010) Glucocorticoid effects on endothelial barrier function in the murine brain endothelia cell line cEND incubated with sera from patients with multiple sclerosis. Mult Scler 16: 293-302. [Crossref]

7. Salvador E, Burek M, Foerster CY (2016) Tight junctions and the Tumor Microenvironment. Curr Pathobiol Rep 4: 135. [Crossref]
8. Forster CY (2008) Tight junctions and the modulation of barrier function in disease. Histochem Cell Biol 130: 55-70. [Crossref]

9. Yamane H, Nakai Y, Takayama M, Iguchi H, Nakagawa T, et al. (1995) Appearance of free radicals in the guinea pig inner ear after noise-induced acoustic trauma. Eur Arch Oto-Rhino-L 252: 504-508. [Crossref]

10. Koo JW, Quintanilla-Dieck L, Jiang M, Liu J, Urdang ZD, et al. (2015) Endotoxemiamediated inflammation potentiates aminoglycoside-induced ototoxicity. Sci Transl Med 7: 298ra118. [Crossref]

11. Inamura N, Salt AN (1992) Permeability changes of the blood-labyrinth barrier measured in vivo during experimental treatments. Hear Res 61: 12-18. [Crossref]

12. Hirose K, Hartsock JJ, Johnson S, Santi P, Salt AN (2014) Systemic lipopolysaccharide compromises the blood-labyrinth barrier and increases entry of serum fluorescein into the perilymph. J Assoc Res Otolaryngol 15: 707-719. [Crossref]

13. Chatterjee S, Lufkin T (2011) The sound of silence: mouse models for hearing loss. Genet Res Int 2011: 416450. [Crossref]

14. Taylor RR, Nevill G, Forge A (2008) Rapid hair cell loss: a mouse model for cochlear lesions. J Assoc Res Otolaryngol 9: 44-64. [Crossref]

15. Suzuki M, Yamasoba T, Ishibashi T, Miller JM, Kaga K (2002) Effect of noise exposure on blood-labyrinth barrier in guinea pigs. Hear Res 164: 12-18. [Crossref]

16. Shi X (2016) Pathophysiology of the cochlear intrastrial fluid-blood barrier (review). Hear Res 338: 52-63. [Crossref]

17. del Zoppo GJ, Hallenbeck JM (2009) Advances in the vascular pathophysiology of ischemic stroke. Thromb Res $98: 73-81$. [Crossref]

18. Heeringa AN, Koppl C (2019) The aging cochlea: Towards unraveling the functional contributions of strial dysfunction and synaptopathy. Hear Res 376: 111-124. [Crossref]

19. Panza F, Lozupone M, Sardone R, Battista P, Piccininni M, et al. (2019) Sensorial frailty: age-related hearing loss and the risk of cognitive impairment and dementia in later life. Ther Adv Chronic Dis 10: 2040622318811000. [Crossref]

Copyright: (C2019 Förster CY. This is an open-access article distributed under the terms of the Creative Commons Attribution License, which permits unrestricted use, distribution, and reproduction in any medium, provided the original author and source are credited. 